\title{
Aquaporin-9 downregulation prevents steatosis in oleic acid-induced non-alcoholic fatty liver disease cell models
}

\author{
CHUAN WANG ${ }^{1}$, ZI-LAN LV ${ }^{2}$, YU-JUN KANG ${ }^{1}$, TING-XIU XIANG ${ }^{1}$, PI-LONG WANG ${ }^{1}$ and ZHENG JIANG ${ }^{1}$ \\ ${ }^{1}$ Department of Gastroenterology, The First Affiliated Hospital, ${ }^{2}$ Key Laboratory of Diagnostic Medicine Designated \\ by the Chinese Ministry of Education, Chongqing Medical University, Chongqing 400016, P.R. China
}

Received June 10,2013; Accepted September 10, 2013

DOI: $10.3892 /$ ijmm.2013.1502

\begin{abstract}
Aquaporin-9 (AQP9) is an aquaglyceroporin that acts as the adipose glycerol channel. However, the role of AQP9 in steatosis in non-alcoholic fatty liver disease (NAFLD) has not yet been fully elucidated. In the present study, the coding sequence of the AQP9 gene was obtained from LO2 cells by RT-PCR, and cloned into the pEGFP-N1 vector. Short hairpin RNA (shRNA) targeting the AQP9 gene was inserted into the pGenesil-1 vector. Recombinant plasmids were confirmed by enzyme digestion and sequence analysis, and transfected into cell models (derived from LO2 cells) of oleic acid-induced NAFLD. Our results demonstrated that AQP9 recombinant plasmids can be effectively expressed in cell models of NAFLD. Furthermore, in comparison with the control group, AQP9 overexpression significantly increased intracellular lipid content, triglyceride (TG), free fatty acid (FFA) and glycerol levels; however, the silencing of AQP9 exerted the opposite effects. Taken together, recombinant plasmids used to induce AQP9 overexpression and to silence AQP9 expression were successfully constructed. AQP9 overexpression aggravated the degree of steatosis; however, the silencing of AQP9 alleviated these effects. Based on these data, we suggest that AQP9 may serve as a novel molecular target for therapeutic intervention in NAFLD.
\end{abstract}

\section{Introduction}

Non-alcoholic fatty liver disease (NAFLD) encompasses a spectrum of liver diseases pertaining to fat accumulation in the liver without significant alcohol consumption. It has become the leading cause of chronic liver injury in the Western world (1). Currently, no effective treatments are available to treat NAFLD; thus, lifestyle modifications via diet and exercise are most commonly recommended (2). Therefore,

Correspondence to: Professor Zheng Jiang, Department of Gastroenterology, The First Affiliated Hospital, Chongqing Medical University, 1 Yixueyuan Road, Chongqing 400016, P.R. China E-mail: jiangz1753@163.com

Key words: aquaporin-9, steatosis, glycerol, non-alcoholic fatty liver disease, cell model there is an urgent need to develop novel treatment options for preventing NAFLD. NAFLD is a clinicohistopathological entity characterized by lipid accumulation in the liver (simple steatosis) (3). In hepatic steatosis, an excess amount of triglycerides (TGs) are acquired and accumulate in hepatocytes. The main sources of TGs are from fatty acids stored in adipose tissue and de novo lipogenesis of fatty acids within the liver (4). Therefore, the role of hepatic glycerol uptake in the development of NAFLD warrants investigation.

Aquaporins (AQPs) are a family of membrane-bound, homotetrameric water channel proteins that allow the movement of water through cell membranes (5). Thus far, 13 AQP subtypes have been identified in human (6). Among these, AQP3, 7, 9 and 10 are subcategorized as aquaglyceroporins which permeabilize glycerol, as well as water (7). AQP9 is expressed in a number of organs, but it is highly expressed in the liver (8). It is thought to be the sole aquaglyceroporin subfamily expressed glycerol channel in liver cells and is localized at the sinusoidal plasma membrane that faces the portal vein (9). However, the role of the AQP9 protein in the pathogenesis of NAFLD remains to be fully elucidated.

In this study, we constructed pEGFP-N1-AQP9 and pGenesil-1-AQP9-short hairpin RNA (shRNA) recombinant eukaryotic expression vectors, which were then transfected into cell models of oleic acid-induced NAFLD, then the expression of AQP9 was detected by RT-PCR and western blot analysis. We demonstrate that transfection with these plasmids can effectively increase and decrease AQP9 expression, respectively. We also demonstrate that high levels of AQP9 protein increase steatosis and that the silencing of AQP9 reverses this effect in cell models of oleic acid-induced NAFLD. Taken together, our results reveal that the downregulation of AQP9 represents a novel potential molecular target for therapeutic intervention in NAFLD.

\section{Materials and methods}

Construction of recombinant pEGFP-N1-AQP9 eukaryotic expression vector. Total RNA was extracted from the LO2 cells using RNAiso Plus (Takara, Dalian, China) and firststrand DNA was synthesized using the RT reagent kit (Takara) with random hexamer primers. The full length AQP9 cDNA was obtained by RT-PCR. The AQP9 nested PCR primer (forward, 5'-GATTTCGGGTTCTAAGTCGC-3' and reverse 
primer, 5'-GAGAATCCCAAACTGACTGC-3'; nested forward, 5'-GGAAGATCTGATGCAGCCTGAGGGAG-3' and reverse, 5'-CGGGGTACCCTGAGTTCATATTTCTC TGG-3') and $\beta$-actin (forward, 5'-ACTGTGCCCATCTAC GAGG-3' and reverse primer, 5'-GAAAGGGTGTAACG CAACTA-3') were synthesized by Takara. The human fulllength AQP9-cDNA was retrieved following the procedures of the E.Z.N.A.Gel Extraction kit (Omega Bio-Tek Inc., Norcross, GA, USA) and ligated to the multi-clony sites of pEGFP-N1 which was digested by $B g l I I$ and $K p n I$ at a ratio of $10: 1$ with T4 DNA ligase (Takara) and incubated at $16^{\circ} \mathrm{C}$ overnight. The ligated product was transformed into competent E. coli $\mathrm{DH} 5 \alpha$. The recombinant plasmid pEGFP-N1-AQP9 was extracted from a single positive clone which was selected with kanamycin, then identified using single digested with $B g l I I$ and double digested with $B g l I I$ and $K p n I$ and the products were evaluated with $1 \%$ agarose gel electrophoresis. The sequence of pEGFPN1-AQP9 was confirmed by Sangon Biotech Co., Ltd. (Shanghai, China).

Construction of recombinant pGenesil-1-AQP9-shRNA eukaryotic expression vector. According to the Homo sapien AQP9 gene sequence (GenBank Accession no. NM_182966) and the principles of shRNA design, a shRNA site targeting the AQP9 gene was selected. The oligonucleotide sequences encoding AQP9 shRNA (forward, 5'-GATCCGCTGTGTC TTTAGCAATGTGTTTCGACACATTGCTAAAGACACAG CTTTTTTA-3' and reverse, 5'-AGCTTAAAAAAGCTGTGT CTTTAGCAATGTGTCGAAACACATTGCTAAAGACACA GCG-3'); and scrambled shRNA (forward, 5'-GATCCG AATCCGCACTACTCCTTACATTCGTGTAAGGAGTAGTG CGGATTCTTTTTTA-3' and reverse, 5'-AGCTTAAAAA AGAATCCGCACTACTCCTTACACGAATGTAAGGAGTA GTGCGGATTCG-3') were synthesized by Invitrogen (Carlsbad, CA, USA); these two pairs of oligonucleotides were annealed into double-stranded DNA, then cloned into the pGenesil-1 vector which was double digested with BamHI and HindIII. These recombinant plasmids were double digested with EcoRI and HindIII for enzyme digestion analysis and sequenced by Sangon Biotech Co., Ltd.

Establishment of oleic acid-induced NAFLD cell models. The 3 rd passage LO2 cells $\left(1 \times 10^{4}\right.$ cells/well) were inoculated into a 96-well plate and cultured in RPMI-1640 supplemented with $10 \%$ fetal bovine serum in $5 \% \mathrm{CO}_{2}$ at $37^{\circ} \mathrm{C}$. The following day, cells were treated with $0,10,20,30,40$ or $50 \mu \mathrm{g} / \mathrm{ml}$ of oleic acid (Sigma-Aldrich Corp., St. Louis, MO, USA) for $72 \mathrm{~h}$, then the culture medium was discarded and the cells were washed three times in phosphate buffer solution, and $20 \mu \mathrm{l}$ of MTT labeling reagent $(0.5 \mathrm{mg} / \mathrm{ml})$ (Sigma-Aldrich Corp.) were added to each well. The plates were then incubated for $4 \mathrm{~h}$ and $150 \mu 1$ of dimethylsulfoxide was added. Optical density values were detected at the $490 \mathrm{~nm}$ wavelength. A cell model of NAFLD was induced by oleic acid. Each assay was performed in octuplicate.

Transfection of the recombinant $p E G F P-N 1-A Q P 9$ and $p G e n-$ esil-1-AQP9-shRNA eukaryotic expression vector into cell models of NAFLD. Endotoxin-free plasmid was extracted using the E.Z.N.A. Endo-Free Plasmid Mini kit I (Omega Bio-Tek
Inc.). Cell models of Oleic acid-induced NAFLD were cultured in RPMI-1640 containing $10 \%$ fetal bovine serum at $37^{\circ} \mathrm{C}$ in a humidified atmosphere of $5 \% \mathrm{CO}_{2}$. Cells were passaged and transfected with endotoxin-free plasmid at $80-90 \%$ confluence using Lipofectamine 2000 reagent (Invitrogen) according to the manufacturer's instructions. Cells were divided into eight groups: untreated, oleic acid, pEGFP-N1-AQP9, pEGFP-N1, pGenesil-1-AQP9-shRNA, pGenesil-1-scrambled-shRNA, pGenesil-1 and double-distilled water $\left(\mathrm{dd}_{2} \mathrm{O}\right)$ group. Seventy-two hours after transfection, the expression of GFP was observed under an inverted fluorescence microscope and the cells were collected to conduct subsequent experiments.

RT-PCR analysis. Total RNA was isolated from each group by RNAiso Plus according to the manufacturer's instructions (Takara). cDNA was prepared using the RT reagent kit and RT-PCR was performed using AQP9 primer (forward, 5'-CTTTGGACGGATGAAATGGTT-3' and reverse, 5'-GAG TCAGGCTCTGGATGGTG-3') and $\beta$-actin was detected as an internal reference. Each assay was performed in duplicate.

Western blot analysis. Total protein was isolated from each group and the protein concentration was detected using the BCA protein assay kit (Beyotime Institute of Biotechnology, Hangzhou, China). Western blot analysis was performed using rabbit anti-human AQP9 polyclonal antibody (Santa Cruz Biotechnology, Inc., Santa Cruz, CA, USA) and rabbit anti-human $\beta$-actin polyclonal antibody (Biosynthesis Biotechnology Co., Ltd., Beijing, China), followed by incubation with HRP-conjugated goat anti-rabbit IgG (Biosynthesis Biotechnology Co., Ltd.). The proteins of interest were detected using BeyoECL plus reagent (Beyotime Institute of Biotechnology) following the manufacturer's instructions. Each assay was performed in duplicate.

Oil red $O$ staining. Briefly, the culture medium was discarded and the cells were washed were washed three times in phosphate buffer solution, then fixed with $4 \%$ paraformaldehyde for $30 \mathrm{~min}$ and stained with $5 \%$ oil red $\mathrm{O}$ solution for $30 \mathrm{~min}$. They were then washed with $60 \%$ isopropanol for $30 \mathrm{sec}$ and then rinsed with $\mathrm{ddH}_{2} \mathrm{O}$ for $30 \mathrm{sec}$, counterstained with hematoxylin for $3 \mathrm{~min}$, rinsed with $\mathrm{ddH}_{2} \mathrm{O}$ for $5 \mathrm{~min}$, fixed with neutral balata, and then observed under an upright microscope. Each assay was performed in triplicate.

Determination of TG, free fatty acid (FFA) and glycerol levels. Briefly, the cells were washed with phosphate buffer solution three times and lysed by repeated freezing and thawing. Intracellular lipid content was determined using the TG assay kit (Beihua Kangtai, Beijing, China), FFA assay kit (Jiancheng Bioengineering Institute, Nanjing, China) and glycerol GPO-POD assay kit (Applygen, Beijing, China) according to the manufacturer's instructions. Each assay was performed in quintuplicate.

Statistical analysis. Statistical analyses were conducted using SPSS 17.0 software. Data are presented as the means \pm standard deviation. One-way ANOVA was conducted to assess differences among groups. A P-value $\leq 0.05$ was considered to indicate a statistically significant difference. 

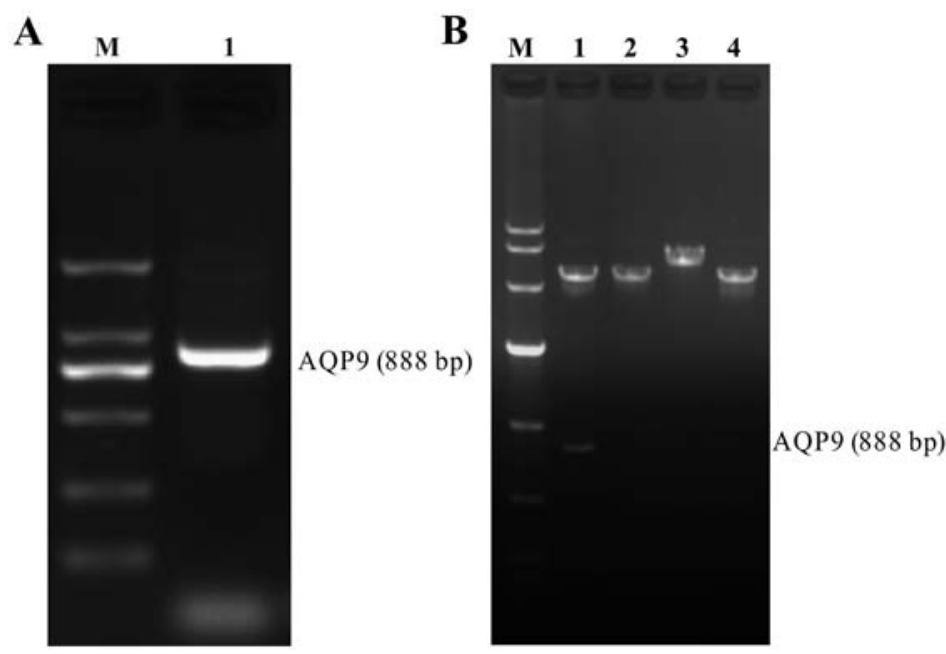

Figure 1. Evaluation of RT-PCR product and recombinant pEGFP-N1-aquaporin-9 (AQP9) eukaryotic expression vector. (A) The full-length AQP9 gene was obtained from LO2 cells by RT-PCR and separated on a 1.5\% agarose gel. M, DL 2,000 DNA marker; lane 1, AQP9 products. (B) The recombinant pEGFPN1-AQP9 vector was single digested with $B g l I I$ and double digested with $B g l I I$ and $K p n I$ and the products were evaluated by $1 \%$ agarose gel electrophoresis. M, DL 10,000 DNA marker; lane 1, pEGFP-N1-AQP9 was double digested with $B g l I I$ and $K p n I$; lane 2, pEGFP-N1 was double digested with $B g l I I$ and $K p n I$; lane 3, pEGFP-N1-AQP9 was single digested with BglII; lane 4, pEGFP-N1 was single digested with $B g l$ II.

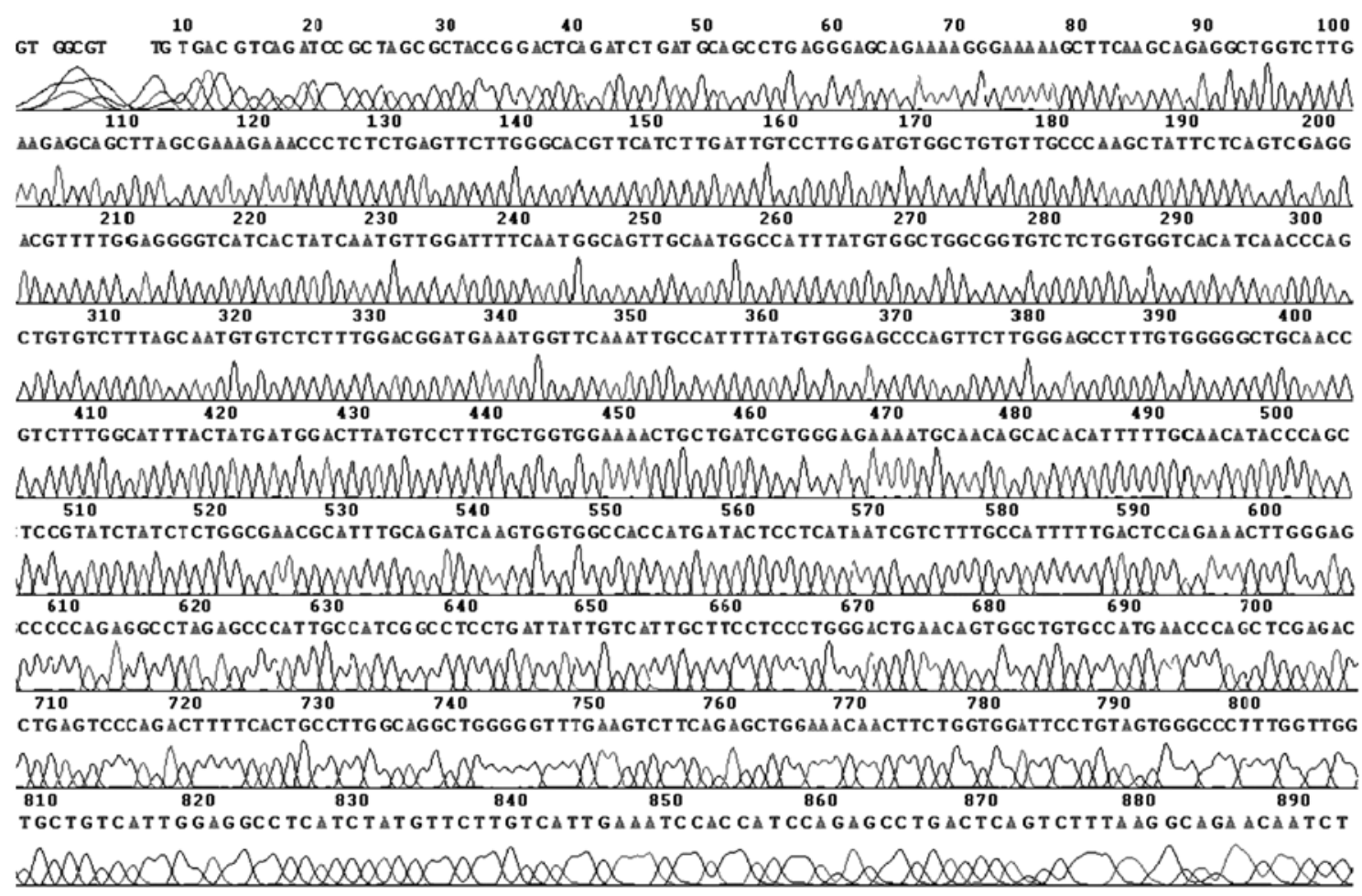

Figure 2. Sequence of full-length 888 bp aquaporin-9 (AQP9) gene.

\section{Results}

Evaluation of RT-PCR product and recombinant pEGFPN1-AQP9 eukaryotic expression vector. The RT-PCR products were loaded on $1.5 \%$ agarose gels, and the band for full-length AQP9 cDNA was located at 888 bp (Fig. 1A). After the AQP9 cDNA fragment was inserted into the pEGFP-N1 plasmid (5428 bp), pEGFP-N1-AQP9 recombinant plasmids were digested by restriction enzymes and separated on a $1 \%$ agarose gel. The positive plasmid was confirmed by DNA sequencing.
Our data demonstrated that after both pEGFP-N1-AQP9 and pEGFP-N1 were single and double digested, separately, the AQP9 band was detected in the double digested pEGFPN1-AQP9, but not in pEGFP-N1, and the band of single digested pEGFP-N1-AQP9 was higher than that of pEGFP-N1 (Fig. 1B). From sequencing analysis, there was a nonsense mutation at site 45: $\mathrm{A} \rightarrow \mathrm{G}$. The sequencing map was as shown in Fig. 2.

Evaluation of recombinant pGenesil-1-AQP9-shRNA eukaryotic expression vector. pGenesil-1-AQP9-shRNA recombinant 


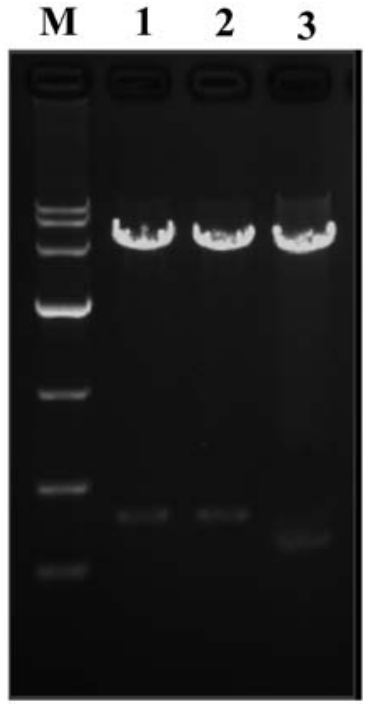

Figure 3. Evaluation of recombinant pGenesil-1-AQP9-shRNA eukaryotic expression vector. Enzyme digestion analysis of pGenesil-1-AQP9-shRNA and pGenesil-1-scrambled shRNA. M, DL 10,000 DNA marker; lane 1, pGenesil-1-AQP9-shRNA was double digested with EcoRI and HindIII; lane 2, pGenesil-1-scrambled shRNA was double digested with EcoRI and HindIII; lane 3, pGenesil-1 was double digested with EcoRI and HindIII. AQP9, aquaporin-9; shRNA, short hairpin RNA.

plasmids were digested by restriction enzymes and evaluated with $1 \%$ agarose gel electrophoresis. The positive plasmid was confirmed by DNA sequencing. The band of double digested and pGenesil-1-scrambled shRNA was higher than that of pGenesil-1 (Fig. 3). Sequencing analysis confirmed that the inserted sequence was consistent with the expected sequence (Fig. 4).

Optimizing the concentration of oleic acid by MTT assay. To determine the optimal concentration of oleic acid for the induction of steatosis, the cell viability of the cell models of oleic acid-induced NAFLD was determined by MTT assay following treatment with oleic acid $(0,10,20,30,40$ or $50 \mu \mathrm{g} /$ $\mathrm{ml}$ ) for $72 \mathrm{~h}$. The cell viability of the cells treated with $>20 \mu \mathrm{g} /$ $\mathrm{ml}$ of oleic acid decreased and significantly decreased when the cells were treated with $50 \mu \mathrm{g} / \mathrm{ml}$ of oleic acid (Fig. 5). Therefore, according to our results, $20 \mu \mathrm{g} / \mathrm{ml}$ of oleic acid was the optimal concentration for inducing steatosis; thus, we selected this dose for the following experiments.
mRNA and protein expression of AQP9 in cell models of oleic acid-induced NAFLD. After the cell models of NAFLD were transfected with recombinant plasmids for $72 \mathrm{~h}$, GFP protein expression was observed under an inverted fluorescence microscope. GFP was observed in the pEGFP-N1-AQP9, pEGFP-N1, pGenesil-1-AQP9-shRNA, pGenesil-1-scrambled shRNA and pGenesil-1 group, but not in the $\mathrm{ddH}_{2} \mathrm{O}$, oleic acid or untreated group (data not shown). To determine the AQP9 mRNA and protein levels in the cell models of oleic acid-induced NAFLD, RT-PCR and western blot analysis were performed. RT-PCR revealed that the delivery of pEGFP-N1-AQP9 and pGenesil-1-AQP9-shRNA resulted in an approximately $70 \%$ overexpression and a $45 \%$ knockdown of mRNA levels compared with the empty vector group, respectively (Fig. 6A). Western blot analysis revealed a corresponding change in AQP9 protein expression (Fig. 6B). These results indicated that the recombinant plasmids were successfully transfected into the cell models of NAFLD and that the vectors, pEGFP-N1-AQP9 and pGenesil-1-AQP9-shRNA, were effective in increasing and suppressing endogenous AQP9 expression, respectively.

Intracellular lipid accumulation is dependent on AQP9 expression in cell models of NAFLD. To detect intracellular lipid accumulation, oil red $\mathrm{O}$ staining was performed. A large number of lipid droplets appeared jacinth with oil red $\mathrm{O}$ staining accompanied by lipid droplet fusion in the pEGFPN1-AQP9 group; a small number of lipid droplets appeared jacinth and lipid droplet fusion was not as evident in the pGenesil-1-AQP9-shRNA group; in the oleic acid group, we also observed lipid droplet fusion, but no lipid droplets were observed in the untreated group (Fig. 7A). These results indicate that oleic acid induces intracellular lipid accumulation in LO2 cells. AQP9 overexpression significantly aggravates intracellular lipid accumulation; however, the silencing of AQP9 alleviates this effect.

Content of TG, FFA and glycerol is depenentd on AQP9 expression in cell models of NAFLD. The content of TG, FFA and glycerol was significantly increased in the oleic acid group, compared with the untreated group (Fig. 7B). Our data also demonstrated that the content of TG, FFA and glycerol was significant increased in the pEGFP-N1-AQP9 group compared with the oleic acid group, while it was significantly decreased in the pGenesil-1-AQP9-shRNA group compared with the oleic acid group. These results suggested that oleic acid increased

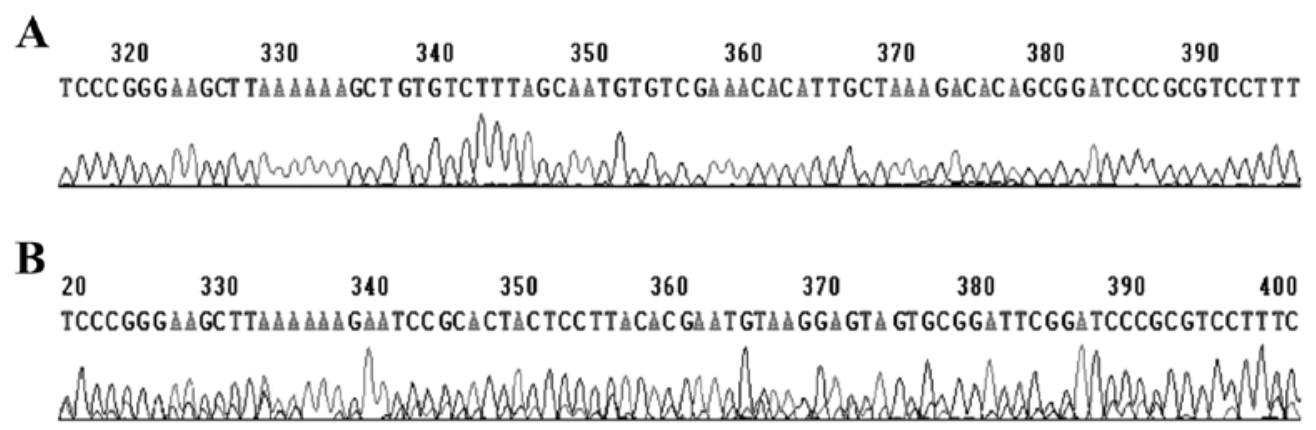

Figure 4. Sequencing map of pGenesil-1-AQP9-shRNA and pGenesil-1-scrambled shRNA. (A) pGenesil-1-AQP9-shRNA; (B) pGenesil-1-scrambled shRNA. AQP9, aquaporin-9; shRNA, short hairpin RNA. 


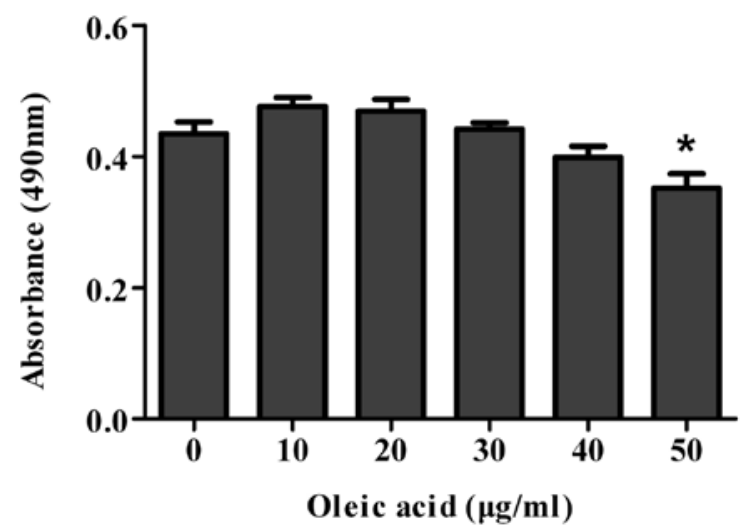

Figure 5. Detecting the optimal concentration of oleic acid by assessing cell viability using MTT Assay. Cell viability was determined by MTT assay after the LO2 cells were treated with $0,10,20,30,40$ or $50 \mu \mathrm{g} / \mathrm{ml}$ of oleic acid ( $\mathrm{P}<0.05$ vs. $0 \mu \mathrm{g} / \mathrm{ml}$ oleic acid group, $\mathrm{n}=8$ ).

the content of TG, FFA and glycerol. AQP9 overexpression induced a significant increase in TG, FFA and glycerol content; however, the silencing of AQP9 significantly reversed this effect. Taken together, these results suggest that AQP9 plays an important role in the progression of hepatic steatosis.

\section{Discussion}

NAFLD consists of a spectrum of pathological states ranging from the hepatic accumulation of lipids known as steatosis to non-alcoholic steatohepatitis, cirrhosis and ultimately, liver failure (10). It occurs in children and adults of all age groups and both genders (11). Its key feature is steatosis in the absence of pathologies, such as viral hepatitis or alcohol abuse (12). By current estimations, NAFLD is perhaps the most common liver disease as it has a prevalence of $6-35 \%$ with a median of $20 \%$ according to a study on populations in several countries (13). NAFLD is particularly associated with obesity, since the prevalence of steatosis is $57.5-74 \%$ in obese subjects from Japan and Italy (14).

Concerning the pathogenesis of NAFLD, the most accepted theory is the 'two hit' hypothesis, in which the first hit involves the accumulation of hepatic TG, which in turn triggers the second hit, inflammation and oxidative stress (15). An extended hypothesis proposed a decrease capacity of hepatic regeneration and the adverse effects of FFA lipotoxicity as the third hit $(16,17)$. These data suggest that alleviating hepatocyte lipid accumulation is an effective therapeutic strategy to prevent NAFLD.

AQP belongs to the major intrinsic protein (MIP) family which can be classified as two types. One type is aquaporin which accounts for the majority of MIPs, including AQP1, AQP2, AQP4, AQP5, AQP6, AQP8, AQP10, AQP11 and AQP12, only permeable to water. The other type is aquaglyceroporin, including AQP3, AQP7, AQP9 and AQP10, permeable to not only water, but also urea, glycerol and even some inorganic ions (18), which play an important role in regulating glycerol transportation and lipid metabolism. AQP9 is a protein channel highly expressed in the liver and is localized at the sinusoidal membrane (19). It is thought to be the sole aquaglyceroporin subfamily expressed glycerol channel in liver cells $(7,9)$.
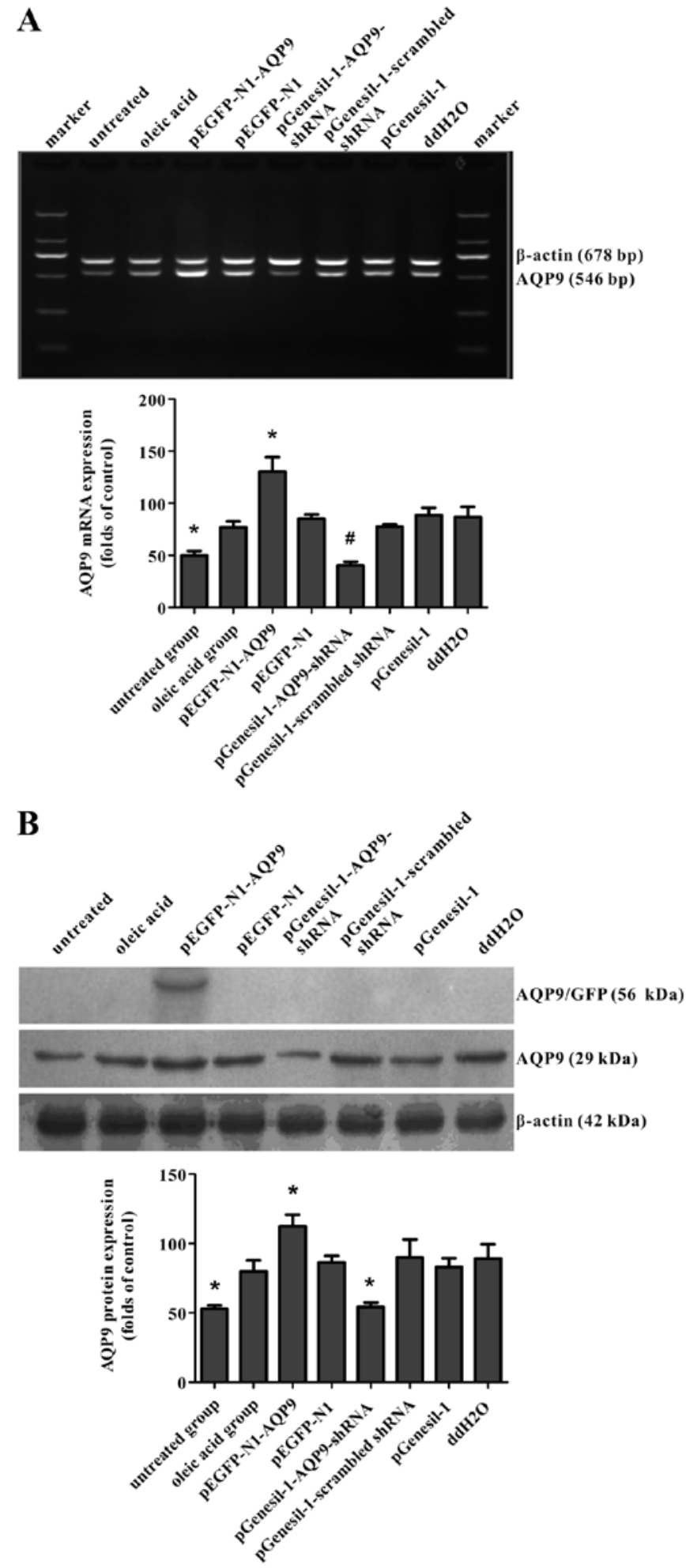

Figure 6. mRNA and protein expression of aquaporin-9 (AQP9) in cell models of oleic acid-induced non-alcoholic fatty liver disease (NAFLD). (A) The expression of AQP9 mRNA was detected by RT-PCR after transfection with pEGFP-N1-AQP9 and pGenesil-1-AQP9-shRNA eukaryotic expression vectors using Lipofectamine 2000 for $72 \mathrm{~h}$. (B) The protein expression of AQP9 was analyzed by western blot analysis following transfection with pEGFPN1-AQP9 and pGenesil-1-AQP9-shRNA eukaryotic expression vectors using Lipofectamine 2000 for $72 \mathrm{~h}\left({ }^{*} \mathrm{P}<0.05\right.$ vs. oleic acid group; ${ }^{\#} \mathrm{P}<0.01$ vs. oleic acid group, $\mathrm{n}=3$ ).

In this study, we used gain- and loss-of-function approaches to determine the role of AQP9 in steatosis in cell models of 
A

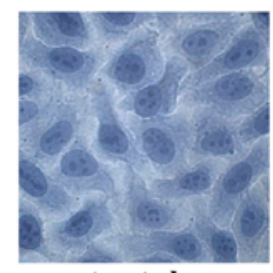

untreated

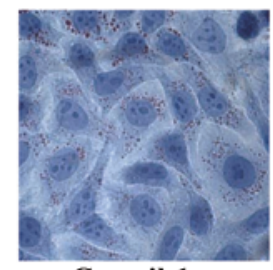

pGenesil-1-

AQP9- shRNA

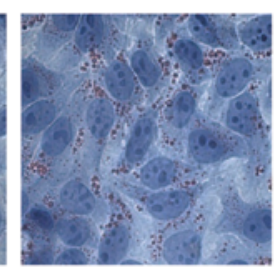

oleic acid

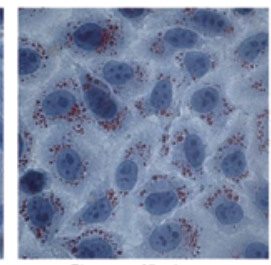

pGenesil-1scrambled shRNA

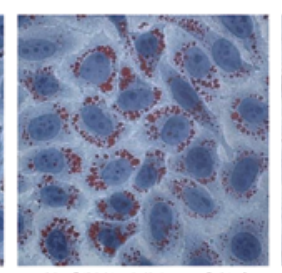

PEGFP-N1-AQP9

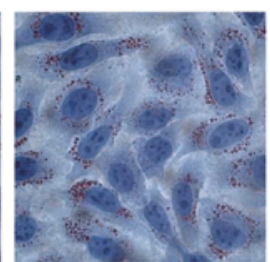

pGenesil-1

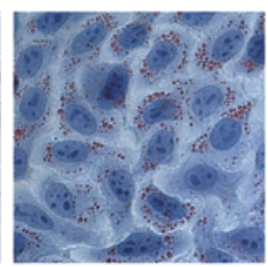

pEGFP-N1

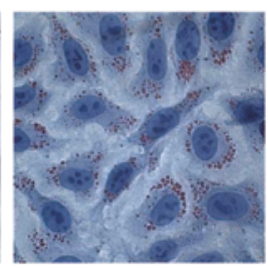

$\mathrm{ddH}_{2} \mathrm{O}$
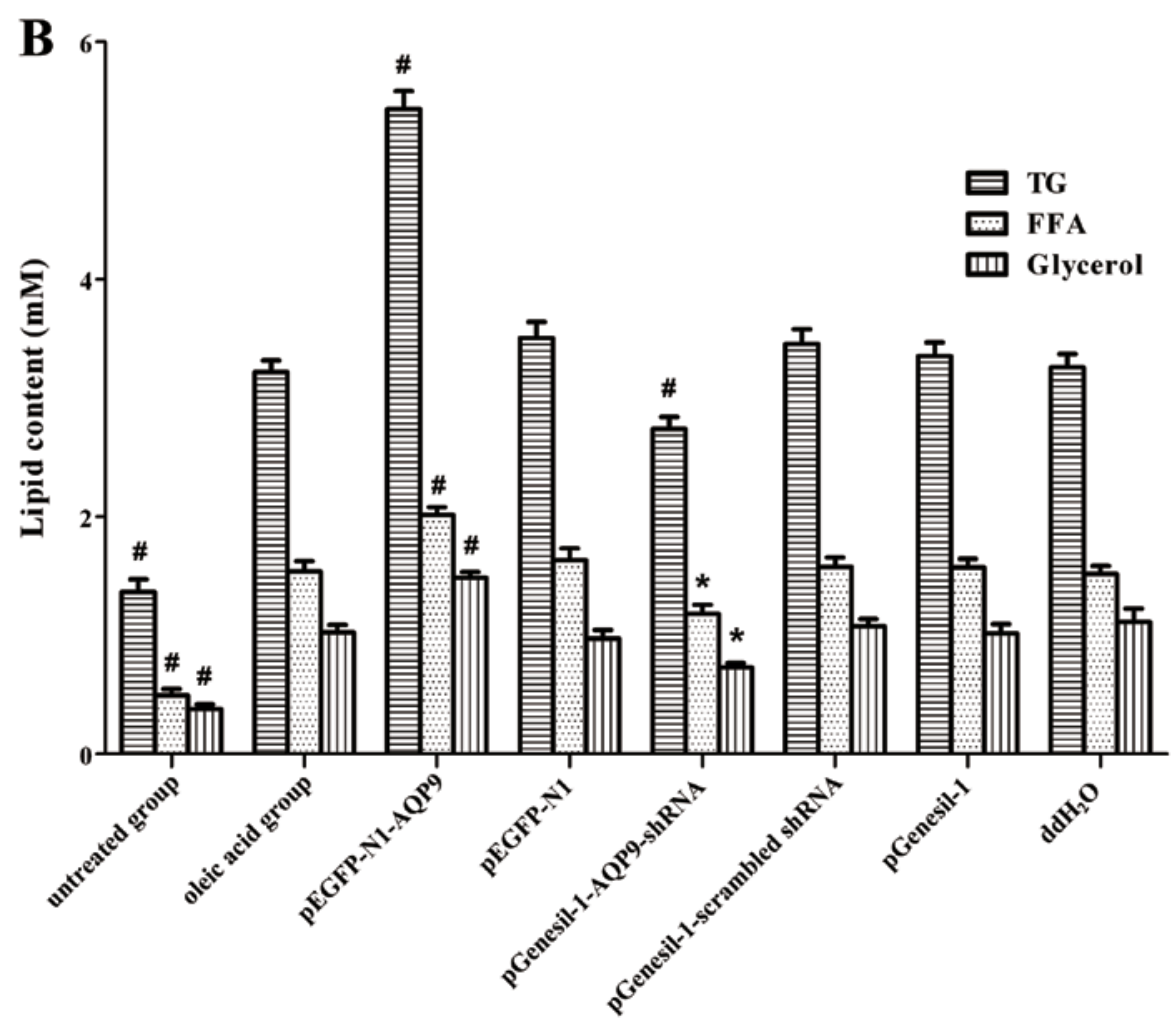

Figure 7. Intracellular lipid accumulation is dependent on aquaporin-9 (AQP9) expression in cell models of oleic acid-induced non-alcoholic fatty liver disease (NAFLD). (A) Oil red O staining. (B) Triglyceride (TG), free fatty acid (FFA) and glycerol content was determined according to the manufacturer's instructions following transfection with oleic pEGFP-N1-AQP9 and pGenesil-1-AQP9-shRNA eukaryotic expression vectors using Lipofectamine 2000 for $72 \mathrm{~h}$ $\left(\right.$ $\mathrm{P}<0.05$ vs. oleic acid group; ${ }^{*} \mathrm{P}<0.01$ vs. oleic acid group, $\left.\mathrm{n}=5\right)$.

oleic acid-induced NAFLD. Our results revealed that AQP9 overexpression significantly increased intracellular lipid content; however, the silencing of AQP9 had the opposite effect. These results are consistent with those from previous studies, showing that an abnormal increase in AQP9 expression leads to a large amount of glycerol entering hepatocytes and increased fat synthesis and accumulation, resulting in the formation of fatty liver $(20,21)$. Glycerol uptake is significantly decreased in cultured astrocytes following transfection with AQP9-small interfering RNA (22). The decreased expression of AQP9 in hepatocytes can effectively block the entry of glycerol into hepatocytes; therefore, targeting AQP9 may be used to prevent and treat NAFLD, which may provide a promising therapeutic strategy for NAFLD. Furthermore, AQP9 acts as an absorption channel for certain drugs. AQP9 has been shown to aid the transfer of the chemotherapeutic drug, arsenic trioxide $(23,24)$. The enforced expression of AQP9 in the tumor cell membrane can effectively enhance the chemotherapeutic efficacy, reducing drug dosage and toxic effects; thus it may provide a novel treatment strategy for tumors $(25,26)$. It has been reported that insulin suppresses AQP9 expression in H4IIE hepatocytes (27). The treatment of rats with streptozotocin (STZ) has been shown to result in a 20 -fold increase in AQP9 expression in the liver, which was blocked by the 
administration of insulin (28). Moreover, insulin resistance is the key factor in the pathogenesis and potential evolution of hepatic steatosis, which is associated with increased peripheral lipolysis with the release of FFA from visceral fat, the hepatic uptake of FFA and hepatocellular TG synthesis and accumulation $(29,30)$. Thus, the knockout of AQP9 may play an important role against insulin resistance.

In conclusion, in this study, we successfully constructed the pEGFP-N1-AQP9 and pGenesil-1-AQP9-shRNA recombinant eukaryotic expression vectors, which were then transfected into cell models (derived from $\mathrm{LO} 2$ cells) of oleic acid-induced NAFLD. Thus, AQP9 expression was effectively increased and suppressed, respectively. In addition, the overexpression of AQP9 significantly increased intracellular lipid content. By contrast, the knockdown of AQP9 exerted the opposite effect. Our findings provide evidence of the potential therapeutic effectiveness of AQP9 in NAFLD. Further studies are required to clarity the molecular mechanisms of action of AQP9 in lipid accumulation in NAFLD.

\section{Acknowledgements}

This study was supported by grants (81070318) from the National NaturalScience Foundation of China(NSFC81070318) and the Medical Research Project from Chongqing Board of Health (YuWeiKeJiao 2010-2-100).

\section{References}

1. Browning JD, Szczepaniak LS, Dobbins R, et al: Prevalence of hepatic steatosis in an urban population in the United States: Impact of ethnicity. Hepatology 40: 1387-1395, 2004.

2. Rodriguez B, Torres DM and Harrison SA: Physical activity: an essential component of lifestyle modification in NAFLD. Nat Rev Gastroenterol Hepatol 9: 726-731, 2012.

3. Fabbrini E, Sullivan S and Klein S: Obesity and nonalcoholic fatty liver disease: biochemical, metabolic, and clinical implications. Hepatology 51: 679-689, 2010.

4. Krawczyk M, Bonfrate L and Portincasa P: Nonalcoholic fatty liver disease. Best Pract Res Clin Gastroenterol 24: 695-708, 2010.

5. Huang HF, He RH, Sun CC, Zhang Y, Meng QX and Ma YY: Function of aquaporins in female and male reproductive systems. Hum Reprod Update 12: 785-795, 2006.

6. Liu H, Zheng Z and Wintour EM: Aquaporins and fetal fluid balance. Placenta 29: 840-847, 2008.

7. Maeda N: Implications of aquaglyceroporins 7 and 9 in glycerol metabolism and metabolic syndrome. Mol Aspects Med 33: 665-675, 2012

8. Elkjaer M, Vajda Z, Nejsum LN, et al: Immunolocalization of AQP9 in liver, epididymis, testis, spleen, and brain. Biochem Biophys Res Commun 276: 1118-1128, 2000.

9. Maeda N, Funahashi T and Shimomura I: Metabolic impact of adipose and hepatic glycerol channels aquaporin 7 and aquaporin 9. Nat Clin Pract Endocrinol Metab 4: 627-634, 2008.
10. Chalasani N, Younossi Z, Lavine JE, et al: The diagnosis and management of non-alcoholic fatty liver disease: practice Guideline by the American Association for the Study of Liver Diseases, American College of Gastroenterology, and the American Gastroenterological Association. Hepatology 55: 2005-2023, 2012.

11. Roberts EA: Pediatric nonalcoholic fatty liver disease (NAFLD): A 'growing' problem? J Hepatol 46: 1133-1142, 2007.

12. Angulo P and Lindor KD: Non-alcoholic fatty liver disease. J Gastroenterol Hepatol 17 (Suppl): S186-S190, 2002.

13. Vernon G, Baranova A and Younossi ZM: Systematic review: the epidemiology and natural history of non-alcoholic fatty liver disease and non-alcoholic steatohepatitis in adults. Aliment Pharmacol Ther 34: 274-285, 2011.

14. Tailleux A, Wouters K and Staels B: Roles of PPARs in NAFLD: potential therapeutic targets. Biochim Biophys Acta 1821: 809-818, 2012.

15. Day CP and James OF: Steatohepatitis: a tale of two 'hits'? Gastroenterology 114: 842-845, 1998.

16. Feldstein AE, Werneburg NW, Canbay A, et al: Free fatty acids promote hepatic lipotoxicity by stimulating TNF-alpha expression via a lysosomal pathway. Hepatology 40: 185-194, 2004.

17. Day CP: From fat to inflammation. Gastroenterology 130: 207-210, 2006.

18. Gomes D, Agasse A, Thiebaud P, Delrot S, Geros H and Chaumont F: Aquaporins are multifunctional water and solute transporters highly divergent in living organisms. Biochim Biophys Acta 1788: 1213-1228, 2009.

19. Carbrey JM, Gorelick-Feldman DA, Kozono D, Praetorius J, Nielsen S and Agre P: Aquaglyceroporin AQP9: solute permeation and metabolic control of expression in liver. Proc Natl Acad Sci USA 100: 2945-2950, 2003.

20. Hara-Chikuma M and Verkman AS: Physiological roles of glycerol-transporting aquaporins: the aquaglyceroporins. Cell Mol Life Sci 63: 1386-1392, 2006.

21. Maeda N, Hibuse T and Funahashi T: Role of aquaporin-7 and aquaporin-9 in glycerol metabolism; involvement in obesity. Handb Exp Pharmacol 190: 233-249, 2009.

22. Badaut J, Brunet JF, Guerin C, Regli L and Pellerin L: Alteration of glucose metabolism in cultured astrocytes after AQP9-small interference RNA application. Brain Res 1473: 19-24, 2012.

23. Hamdi M, Sanchez MA, Beene LC, et al: Arsenic transport by zebrafish aquaglyceroporins. BMC Mol Biol 10: 104, 2009.

24. Drobna Z, Walton FS, Paul DS, Xing W, Thomas DJ and Styblo M: Metabolism of arsenic in human liver: the role of membrane transporters. Arch Toxicol 84: 3-16, 2010.

25. Leung J, Pang A, Yuen WH, Kwong YL and Tse EWC: Relationship of expression of aquaglyceroporin 9 with arsenic uptake and sensitivity in leukemia cells. Blood 109: 740-746, 2007.

26. Gao L, Gao Y, Li X, et al: Aquaporins mediate the chemoresistance of human melanoma cells to arsenite. Mol Oncol 6: 81-87, 2012.

27. Hibuse T, Maeda N, Nagasawa A and Funahashi T: Aquaporins and glycerol metabolism. Biochim Biophys Acta 1758: 1004-1011, 2006.

28. King LS, Kozono D and Agre P: From structure to disease: the evolving tale of aquaporin biology. Nat Rev Mol Cell Biol 5: 687-698, 2004

29. Parekh S and Anania FA: Abnormal lipid and glucose metabolism in obesity: implications for nonalcoholic fatty liver disease. Gastroenterology 132: 2191-2207, 2007.

30. Shoelson SE, Herrero L and Naaz A: Obesity, inflammation, and insulin resistance. Gastroenterology 132: 2169-2180, 2007. 